\title{
A high concentration of prorenin in early pregnancy is associated with development of pre-eclampsia in women with type 1 diabetes
}

\author{
L. Ringholm • U. Pedersen-Bjergaard • \\ B. Thorsteinsson • F. Boomsma • P. Damm • \\ E. R. Mathiesen
}

Received: 8 November 2010 / Accepted: 24 January 2011 / Published online: 22 February 2011

(C) Springer-Verlag 2011

\begin{abstract}
Aims/hypothesis The aim of this study was to investigate whether components of the renin-angiotensin system and semicarbazide-sensitive amine oxidase (SSAO) are associated with the development of pre-eclampsia in women with type 1 diabetes.

Methods This was an observational study of 107 consecutive pregnant women with type 1 diabetes (median duration 16 years [range 1-36 years], $\mathrm{HbA}_{1 \mathrm{c}} 6.6 \%$ [range $4.9-10.5 \%$ ]) in early pregnancy. At 8, 14, 21, 27 and 33 weeks and once within 5 days postpartum, blood was sampled for measurements of
\end{abstract}

L. Ringholm $(\bowtie) \cdot$ P. Damm $\cdot$ E. R. Mathiesen

Center for Pregnant Women with Diabetes, Rigshospitalet,

University of Copenhagen,

Blegdamsvej 9,

2100 Copenhagen, Denmark

e-mail: enel@dadlnet.dk

L. Ringholm $\cdot$ E. R. Mathiesen

Department of Endocrinology, Rigshospitalet,

Copenhagen, Denmark

U. Pedersen-Bjergaard $\cdot$ B. Thorsteinsson

Endocrinology Section,

Department of Cardiology and Endocrinology, Hillerød Hospital,

Hillerød, Denmark

B. Thorsteinsson · P. Damm • E. R. Mathiesen

Faculty of Health Sciences, University of Copenhagen,

Copenhagen, Denmark

F. Boomsma

Section of Vascular Pharmacology and Metabolism,

Department of Internal Medicine, Erasmus Medical Center,

Rotterdam, the Netherlands

P. Damm

Department of Obstetrics, Rigshospitalet,

Copenhagen, Denmark prorenin, renin, angiotensinogen, $\mathrm{ACE}$ and $\mathrm{SSAO} . \mathrm{HbA}_{1 \mathrm{c}}$, blood pressure and urinary albumin excretion were recorded. Pre-eclampsia was defined as blood pressure $>140 / 90 \mathrm{mmHg}$ and proteinuria $\geq 300 \mathrm{mg} / 24 \mathrm{~h}$ after 20 weeks.

Results Pre-eclampsia developed in nine women (8\%) with longer diabetes duration (median 20 [range 10-32] vs 16 [range 1-36] years, $p=0.04$ ), higher SSAO concentrations (592 [range 372-914] vs 522 [range 264-872] mU/l, $p=0.04)$ and a tendency towards higher prorenin levels (136 [range 50-296] vs 101 [range 21-316] ng angiotensin I $\mathrm{ml}^{-1} \mathrm{~h}^{-1}, p=0.06$ ) at 8 weeks compared with women without pre-eclampsia. Levels of renin, angiotensinogen and ACE did not differ in the two groups. Throughout pregnancy, prorenin and SSAO levels were $30 \%(p=0.004)$ and $16 \%(p=0.04)$ higher, respectively, in women developing pre-eclampsia. Using multivariate logistic regression analysis, prorenin concentration at 8 weeks was associated with pre-eclampsia (OR 4.4 [95\% CI 1.5-13.0], $p=0.007$ ), i.e. an increase of prorenin of $100 \mathrm{ng}$ angiotensin $\mathrm{I} \mathrm{ml}^{-1} \mathrm{~h}^{-1}$ implies a 4.4 times higher risk of subsequent pre-eclampsia. Conclusions/interpretation In type 1 diabetic women with pre-eclampsia, a higher concentration of prorenin in early pregnancy and higher levels of prorenin and SSAO throughout pregnancy were seen.

Keywords ACE - Angiotensinogen - Antihypertensive therapy · Pre-eclampsia · Pregnancy · Prorenin · Renin . Renin-angiotensin system · SSAO - Type 1 diabetes
Abbreviations
AI Angiotensin I
SMPG Self-monitored plasma glucose
SSAO Semicarbazide-sensitive amine oxidase
UAE Urinary albumin excretion 


\section{Introduction}

In women with type 1 diabetes, microalbuminuria and high blood pressure levels are risk factors for pre-eclampsia, but even diabetic women with normal blood pressure and normal urinary albumin excretion (UAE) are at risk of developing pre-eclampsia [1]. During the early stages of normal pregnancy, the renin-angiotensin system is activated with a tenfold increase in plasma prorenin levels at three to six gestational weeks, whereas much lower prorenin levels are detected from 9 weeks onwards, consistent with a role for the renin-angiotensin system in placentation and embryonic development [2].

In pre-eclampsia, perturbation of the renin-angiotensin system is seen with increased vascular responsiveness to angiotensin II [3]. It is unknown whether other components of the renin-angiotensin system are affected in early pregnancy among women with type 1 diabetes who subsequently develop pre-eclampsia and whether this is affected by antihypertensive therapy.

Semicarbazide-sensitive amine oxidase (SSAO), an adhesion molecule present in blood vessels and plasma, is associated with endothelial cell cytotoxicity [4] and may be a marker of endothelial dysfunction and pre-eclampsia.

In this study, we investigated whether circulating concentrations of components of the renin-angiotensin system and of SSAO are associated with the development of pre-eclampsia in pregnant women with type 1 diabetes.

\section{Methods}

In a prospective observational study we consecutively included 107 Danish-speaking Europid women with type 1 diabetes referred to the Center for Pregnant Women with Diabetes, Rigshospitalet, before 14 completed gestational weeks with a single living fetus, from September 2004 to August 2006 [5].

The research protocol was approved by the regional ethics committees.

Blood sampling was performed at medians of 8 (range 513), 14 (12-16), 21 (20-23), 27 (25-29) and 33 (31-35) weeks and once within 5 days postpartum [5]. Concentrations of prorenin and angiotensinogen and activity of renin, ACE and SSAO were analysed as previously described $[5,6]$. Prorenin concentration was given as nanograms angiotensin I per millilitre per hour (ng AI ml ${ }^{-1} \mathrm{~h}^{-1}$ ). For all assays, intra- and interassay CVs were below $10 \%[5,6]$.

Routine self-monitored plasma glucose (SMPG) was recommended at least seven times daily to obtain preprandial SMPG of 4.0-6.0 mmol/1, $90 \mathrm{~min}$ postprandial SMPG of $4.0-8.0 \mathrm{mmol} / \mathrm{l}$, pre-bedtime SMPG of $6.0-8.0 \mathrm{mmol} / \mathrm{l}$ and $\mathrm{HbA}_{1 \mathrm{c}} \leq 5.6 \%$, in late pregnancy. Weight, $\mathrm{HbA}_{1 \mathrm{c}}$, insulin dose and blood pressure were recorded at each clinical consultation at 1 or 2 week intervals throughout pregnancy [5].

Presence of diabetic retinopathy at inclusion was routinely assessed by photo screening, which was evaluated by one experienced ophthalmologist [7].

Based on $24 \mathrm{~h} \mathrm{UAE}$ at inclusion, the women were classified as having normoalbuminuria (UAE $<30 \mathrm{mg} / 24 \mathrm{~h}$ ), microalbuminuria (UAE 30-299 $\mathrm{mg} / 24 \mathrm{~h}$ ) or diabetic nephropathy (UAE $\geq 300 \mathrm{mg} / 24 \mathrm{~h}$ ). Pre-eclampsia in women with normoalbuminuria or microalbuminuria was defined as blood pressure $>140 / 90 \mathrm{mmHg}$ accompanied by proteinuria $(\geq 1+)$ using a sterile urinary dip-stick (Uristix, Bayer Diagnostics, Bridgend, UK) or proteinuria $\geq 300 \mathrm{mg} / 24 \mathrm{~h}$ later than 20 weeks. In women with diabetic nephropathy, the diagnosis was based on the same findings accompanied by a sudden increase of $\geq 15 \%$ in systolic or diastolic blood pressure. Antihypertensive therapy was initiated if blood

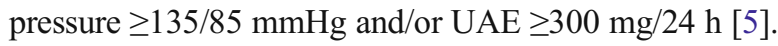

Statistical analyses Data are given as median (range) or numbers (\%). Categorical variables were compared by $\chi^{2}$ or Fisher's exact test, as appropriate. Continuous variables were analysed by parametric or non-parametric tests. Loglinear correlation analyses were performed using Pearson's coefficient, denoted $r$ [5].

To identify associations with pre-eclampsia, univariate logistic regression analysis was conducted with pre-eclampsia during pregnancy (yes/no) as dependent variable. Significant associations were further tested in backward stepwise multivariate logistic regression analysis. A variable remaining significant in this analysis was considered to be significantly associated with the development of pre-eclampsia.

Statistically significant differences were defined as $p<0.05$.

\section{Results}

Pre-eclampsia developed in nine women (8\%) at 34 (33-36) weeks (Table 1).

Throughout pregnancy, prorenin concentrations and SSAO activity remained $30 \%(p=0.004)$ and $16 \%$ $(p=0.04)$ higher, respectively, in women developing preeclampsia. No women with prorenin concentrations below $50.2 \mathrm{ng} \mathrm{AI} \mathrm{ml} l^{-1} \mathrm{~h}^{-1}$ or SSAO activity below $372 \mathrm{mU} / 1$ at inclusion developed pre-eclampsia (Table 1).

Prorenin concentrations decreased slightly and similarly from 8 weeks until postpartum in women with and without pre-eclampsia. Angiotensinogen concentrations and activity of renin and ACE were comparable between women with and without pre-eclampsia ( $p=0.79, p=0.43$ and $p=0.51$, respectively, data not shown).

At inclusion, prorenin concentrations were positively correlated with duration of diabetes $(r=0.33, p=0.0007)$. 
Table 1 Baseline data at inclusion and pregnancy outcome in 107 women with type 1 diabetes according to development of pre-eclampsia during pregnancy

\begin{tabular}{|c|c|c|c|}
\hline Variable & $\begin{array}{l}\text { Women without pre-eclampsia } \\
\text { in pregnancy }\end{array}$ & $\begin{array}{l}\text { Women with pre-eclampsia } \\
\text { in pregnancy }{ }^{\mathrm{b}}\end{array}$ & $p$ value \\
\hline Age (years) & $30(21-42)$ & $31(25-39)$ & 0.44 \\
\hline Duration of diabetes (years) & $16(1-36)$ & $20(10-32)$ & 0.04 \\
\hline Gestational age (days) & $61.5(37-94)$ & $57(47-82)$ & 0.40 \\
\hline $\mathrm{HbA}_{1 \mathrm{c}}(\%)$ & $6.6(4.9-10.5)$ & $6.7(5.7-8.5)$ & 0.37 \\
\hline Insulin dose $(\mathrm{U} / \mathrm{kg})$ & $0.76(0.3-1.7)$ & $0.88(0.6-1.1)$ & 0.33 \\
\hline BMI before pregnancy $\left(\mathrm{kg} / \mathrm{m}^{2}\right)$ & $24.4(17.3-43.8)$ & $24.0(20.1-32.4)$ & 0.49 \\
\hline Insulin type (human insulin/insulin analogues), $n(\%)$ & $52(53) / 46(47)$ & $8(89) / 1(11)$ & 0.07 \\
\hline Diabetic retinopathy $^{\mathrm{c}}, n(\%)$ & $52(56)$ & $8(100)$ & 0.02 \\
\hline Microalbuminuria/diabetic nephropathy, $n(\%)$ & $10(10) / 3(3)$ & $0 / 3(33)$ & 0.01 \\
\hline Antihypertensive treatment, $n(\%)$ & $11(11)$ & $3(33)$ & 0.09 \\
\hline $\begin{array}{l}\text { Number of women initiating antihypertensive therapy } \\
\text { during pregnancy, } n(\%)\end{array}$ & $4(4)$ & $6(67)$ & $<0.0001$ \\
\hline Systolic blood pressure $(\mathrm{mmHg})$ & $119(88-150)$ & $125(100-150)$ & 0.94 \\
\hline Diastolic blood pressure $(\mathrm{mmHg})$ & $70(55-86)$ & $71(60-85)$ & 0.68 \\
\hline Prorenin concentration $\left(\mathrm{ng} \mathrm{AI} \mathrm{ml} \mathrm{Al}^{-1} \mathrm{~h}^{-1}\right.$ ) & $101.1(21.1-315.6)$ & $136.0(50.2-296.0)$ & 0.06 \\
\hline Renin activity (ng $\mathrm{AI} \mathrm{ml} l^{-1} \mathrm{~h}^{-1}$ ) & $7.2(2.0-20.0)$ & $6.7(3.6-16.0)$ & 0.90 \\
\hline Angiotensinogen concentration $(\mathrm{pmol} / \mathrm{ml})$ & $2,114(744-4,633)$ & $2,214(931-4,108)$ & 0.73 \\
\hline ACE activity (U/l) & $15.6(7.8-26.5)$ & $14.4(4.8-26.5)$ & 0.91 \\
\hline SSAO activity $(\mathrm{mU} / \mathrm{l})$ & $522(264-872)$ & $592(372-914)$ & 0.04 \\
\hline Creatinine $(\mu \mathrm{mol} / \mathrm{l})$ & $51(33-79)$ & $52(42-95)$ & 0.67 \\
\hline Parity $(n)$ & $2(1-4)$ & $1(1-3)$ & 0.71 \\
\hline Preterm delivery ( $<37$ gestational weeks), $n(\%)$ & $16(16)$ & $7(78)$ & $<0.0001$ \\
\hline Birthweight $z$ score (SD units) & $1.04(-1.51-5.17)$ & $1.62(-1.94-7.05)$ & 0.91 \\
\hline Small for gestational age infants $(<10$ th centile), $n(\%)$ & $1(1)$ & $2(22)$ & 0.02 \\
\hline Large for gestational age infants ( $>90$ th centile), $n(\%)$ & $45(46)$ & $5(56)$ & 0.73 \\
\hline
\end{tabular}

Data are number (\%) or median (range)

${ }^{\mathrm{a}} n=98(92 \%) ;{ }^{\mathrm{b}} n=9(8 \%) ;{ }^{\mathrm{c}} n=101$, of which there were 93 women without pre-eclampsia and eight women with pre-eclampsia during pregnancy

Throughout pregnancy, activity of ACE and SSAO were positively correlated $(r=0.32, p=0.001$ at 8 weeks; $r=0.38$, $p=0.0002$ at 14 weeks; $r=0.35, p=0.0005$ at 21 weeks; $r=0.37, p=0.0002$ at 27 weeks; $r=0.34, p=0.0007$ at 33 weeks).

Eighty-two women remained normotensive without the need for antihypertensive therapy during pregnancy. In six women developing pre-eclampsia, antihypertensive therapy was initiated at a median of 31.5 weeks (range 14-36). Another four women without pre-eclampsia initiated antihypertensive therapy at 16.5 weeks (range 8-27) because of proteinuria or high blood pressure. One woman discontinued antihypertensive therapy at inclusion because of normal blood pressure and UAE. Among 14 women using antihypertensive therapy during the entire pregnancy, three women developed pre-eclampsia.

Among the six normotensive women with subsequent initiation of antihypertensive therapy and development of pre-eclampsia, prorenin concentrations at 8 weeks were higher $(p=0.03$; Table 2), and throughout pregnancy prorenin concentrations remained $36 \%$ higher $(p=0.001)$ compared with the 82 women remaining normotensive without antihypertensive therapy during pregnancy. No differences were detected in angiotensinogen concentrations $(p=0.51)$ or in activity of renin, ACE or SSAO ( $p=0.57, p=0.96$ and $p=0.64$, respectively).

Using univariate logistic regression analysis including all 107 women, prorenin concentrations at 8 weeks (OR 4.4 [95\% CI 1.5-13.0], $p=0.007$ ), UAE (categorised as $<30$, $30-299$ and $\geq 300 \mathrm{mg} / 24 \mathrm{~h}$ ) at 8 weeks (OR $3.1[95 \% \mathrm{CI}$ $1.3-7.7], p=0.01$ ), duration of diabetes (OR 2.6 per 10 years increase [95\% CI 1.03-6.7], $p=0.04$ ) and SSAO activity at 8 weeks (OR 1.8 per $100 \mathrm{mU} / 1$ increase [95\% CI 1.1-3.1], $p=0.01$ ) were associated with development of pre-eclampsia. Maternal age, gestational age at first blood sampling, prepregnancy BMI, $\mathrm{HbA}_{1 \mathrm{c}}(\%)$, insulin dose $(\mathrm{U} / \mathrm{kg})$, UAE (per 
Table 2 Baseline data at inclusion and pregnancy outcome in 88 type 1 diabetic women with normal blood pressure and no antihypertensive therapy in early pregnancy

\begin{tabular}{|c|c|c|c|}
\hline Variable & $\begin{array}{l}\text { Women without pre-eclampsia } \\
\text { in pregnancy }\end{array}$ & $\begin{array}{l}\text { Women with pre-eclampsia } \\
\text { in pregnancy }\end{array}$ & $p$ value \\
\hline Duration of diabetes (years) & $14(1-36)$ & $19(10-26)$ & 0.13 \\
\hline Gestational age (days) & $61.5(43-94)$ & $52(47-82)$ & 0.11 \\
\hline $\mathrm{HbA}_{1 \mathrm{c}}(\%)$ & $6.6(4.9-10.5)$ & $7.0(6.5-8.5)$ & 0.12 \\
\hline $\mathrm{HbA}_{1 \mathrm{c}}$ at 33 weeks $(\%)$ & $5.9(5.0-7.3)$ & $6.5(5.8-6.7)$ & 0.07 \\
\hline Systolic blood pressure (mmHg) & $118(88-136)$ & $116(100-139)$ & 0.90 \\
\hline Diastolic blood pressure (mmHg) & $70(55-80)$ & $71(60-85)$ & 0.89 \\
\hline Microalbuminuria/diabetic nephropathy, $n(\%)$ & $5(6 \%) / 0$ & $0 / 1(17 \%)$ & 0.10 \\
\hline Prorenin concentration (ng AI ml ${ }^{-1} \mathrm{~h}^{-1}$ ) & $98.1(21.1-315.6)$ & $143.3(82.0-293.0)$ & 0.03 \\
\hline Renin activity (ng AI ml ${ }^{-1} \mathrm{~h}^{-1}$ ) & $7.2(2.0-20.0)$ & $6.5(3.6-16.0)$ & 0.59 \\
\hline Angiotensinogen concentration $(\mathrm{pmol} / \mathrm{ml})$ & $2,166(744-4,498)$ & $2,257(1,594-4,108)$ & 0.70 \\
\hline ACE activity $(\mathrm{U} / \mathrm{l})$ & $15.6(8.6-26.5)$ & $14.1(10.2-18.6)$ & 0.60 \\
\hline SSAO activity (mU/l) & $520(264-872)$ & $556(372-700)$ & 0.54 \\
\hline Creatinine $(\mu \mathrm{mol} / \mathrm{l})$ & $51(33-75)$ & $52(42-55)$ & 0.69 \\
\hline Parity $(n)$ & $2(1-4)$ & $1.5(1-3)$ & 0.83 \\
\hline Gestational age at delivery (days) & $266(241-276)$ & $256(244-262)$ & 0.0007 \\
\hline Preterm delivery $(<37$ gestational weeks), $n(\%)$ & $8(10)$ & $5(83)$ & $<0.0001$ \\
\hline Birthweight $z$ score (SD units) & $1.23(-1.51-5.17)$ & $2.16(-1.30-7.05)$ & 0.24 \\
\hline
\end{tabular}

Data are number $(\%)$ or median (range)

Eighty-two women remained normotensive without antihypertensive therapy during pregnancy. Six women developing pre-eclampsia initiated antihypertensive therapy at median 31.5 weeks (range 14-36)

${ }^{\mathrm{a}} n=82 ;{ }^{\mathrm{b}} n=6$

$100 \mathrm{mg}$ increase/24 h), systolic or diastolic blood pressure, presence of diabetic retinopathy, parity, concentration of angiotensinogen or activity of renin or ACE were not associated with development of pre-eclampsia.

Using multivariate logistic regression analysis including prorenin concentrations at 8 weeks, SSAO activity at 8 weeks, UAE at 8 weeks (categorised as $<30,30-299$ and $\geq 300 \mathrm{mg} / 24 \mathrm{~h}$ ) and duration of diabetes, the only independent predictor of pre-eclampsia among all women was prorenin concentration at 8 weeks (OR 4.4 [95\% CI $1.5-13.0], p=0.007)$, i.e. an increase of prorenin of $100 \mathrm{ng} \mathrm{AI} \mathrm{ml}{ }^{-1} \mathrm{~h}^{-1}$ implies a 4.4 times higher risk of developing pre-eclampsia.

\section{Discussion}

In this observational study of 107 women with type 1 diabetes, high prorenin concentrations in early pregnancy predicted the development of pre-eclampsia in late pregnancy and, throughout pregnancy, prorenin concentrations remained 30\% higher in women developing pre-eclampsia.

To exclude the possible influence of antihypertensive therapy on the components of the renin-angiotensin system and SSAO in early pregnancy, normotensive women without antihypertensive therapy in pregnancy were compared with normotensive women who were without antihypertensive therapy at inclusion and subsequently initiated antihypertensive therapy and developed preeclampsia. Despite the low number of women with preeclampsia in this analysis, a significant difference in prorenin concentrations was detected in early pregnancy compared with women without antihypertensive therapy during pregnancy, while baseline blood pressure and $\mathrm{HbA}_{1 \mathrm{c}}$ were comparable.

Throughout pregnancy, prorenin concentrations remained higher in all six women developing pre-eclampsia as compared with the 82 women remaining normotensive without antihypertensive therapy during pregnancy. This further indicates that high prorenin concentrations are associated with the development of pre-eclampsia in women with type 1 diabetes.

These findings are reminiscent of previous studies in non-pregnant participants showing that increased prorenin concentrations are present in diabetic individuals who subsequently develop microalbuminuria [8]. Pre-eclampsia is thought to be preceded by abnormal placentation, vascular development and trophoblast invasion in early pregnancy. Strongly increased angiotensin II type 1 (AT1) receptor levels have been found in non-diabetic women 
with pre-eclampsia [3]. In non-pregnant diabetic individuals with microvascular complications including microalbuminuria or diabetic nephropathy, changes in the renin-angiotensin system are seen, particularly in terms of increased plasma concentrations of prorenin being disproportionately increased compared with renin [8]. Likewise, microalbuminuria is known to be associated with the development of preeclampsia in women with type 1 diabetes [1]. We speculate that the changes in the renin-angiotensin system observed in the current study reflect maternal susceptibility present before pregnancy.

SSAO activity was higher in early pregnancy and remained $16 \%$ higher throughout pregnancy in women with subsequent pre-eclampsia. Throughout pregnancy, SSAO was positively correlated with ACE activity in agreement with findings in non-pregnant type 1 diabetic individuals, suggesting an association between SSAO and the renin-angiotensin system [9]. Sikkema et al. [4] previously found no association between SSAO and preeclampsia, but in that study, SSAO activity was only assessed in late pregnancy in 14 non-diabetic women with pre-eclampsia and 14 healthy pregnant women.

According to our strategy of strict antihypertensive therapy [5], antihypertensive therapy was given to $23 \%$ of the women. Although the number of women with preeclampsia in this observational study is too small for firm conclusions, a combined effect of improved glycaemic control and early intensive antihypertensive intervention may explain the lower proportion of women with preeclampsia as compared with previous reports of preeclampsia in approximately $13 \%$ of diabetic women [10].

\footnotetext{
Acknowledgements We are indebted to registered nurses E. Stage and C. Barfred, Center for Pregnant Women with Diabetes, Rigshospitalet, Denmark, and laboratory technicians K.M. Larsen and M. Wahl, Center for Pregnant Women with Diabetes, Rigshospitalet, Denmark, for collecting patient data including blood samples. We thank laboratory technicians U. Bhaggoe, A. Bouhuizen and R. de Bruin, Section of Vascular Pharmacology and Metabolism, Department of Internal Medicine, Erasmus Medical Center, Rotterdam, the Netherlands, for performing the analyses of the renin-angiotensin system components and of SSAO.
}

Duality of interest statement The study was supported by unrestricted grants from The Danish Diabetes Association and Novo Nordisk A/S, Bagsværd, Denmark. The authors declare that there are no other potential conflicts of interest relevant to this study.

\section{References}

1. Ekbom P, Damm P, Feldt-Rasmussen B, Feldt-Rasmussen U, Molvig J, Mathiesen ER (2001) Pregnancy outcome in type 1 diabetic women with microalbuminuria. Diabetes Care 24:1739-1744

2. Itskovitz J, Rubattu S, Levron J, Sealey JE (1992) Highest concentrations of prorenin and human chorionic gonadotropin in gestational sacs during early human pregnancy. J Clin Endocrinol Metab 75:906-910

3. Herse F, Dechend R, Harsem NK et al (2007) Dysregulation of the circulating and tissue-based renin-angiotensin system in preeclampsia. Hypertension 49:604-611

4. Sikkema JM, Franx A, Fijnheer R, Nikkels PG, Bruinse HW, Boomsma F (2002) Semicarbazide-sensitive amine oxidase in preeclampsia: no relation with markers of endothelial cell activation. Clin Chim Acta 324:31-38

5. Nielsen LR, Pedersen-Bjergaard U, Thorsteinsson B, Boomsma F, Damm P, Mathiesen ER (2009) Severe hypoglycaemia during pregnancy in women with type 1 diabetes: possible role of renin-angiotensin system activity? Diabetes Res Clin Pract 84:61-67

6. van Dijk J, Boomsma F, Alberts G, Man in’t Veld AJ, Schalekamp MA (1995) Determination of semicarbazide-sensitive amine oxidase activity in human plasma by high-performance liquid chromatography with fluorimetric detection. J Chromatogr B Biomed Appl 663:43-50

7. Vestgaard M, Ringholm L, Laugesen CS, Rasmussen KL, Damm P, Mathiesen ER (2010) Pregnancy-induced sight-threatening diabetic retinopathy in women with type 1 diabetes. Diabet Med 27:431-435

8. Deinum J, Ronn B, Mathiesen E, Derkx FH, Hop WC, Schalekamp MA (1999) Increase in serum prorenin precedes onset of microalbuminuria in patients with insulin-dependent diabetes mellitus. Diabetologia 42:1006-1010

9. Boomsma F, Pedersen-Bjergaard U, Agerholm-Larsen B et al (2005) Association between plasma activities of semicarbazidesensitive amine oxidase and angiotensin-converting enzyme in patients with type 1 diabetes mellitus. Diabetologia 48:1002-1007

10. Hiilesmaa V, Suhonen L, Teramo K (2000) Glycaemic control is associated with pre-eclampsia but not with pregnancy-induced hypertension in women with type I diabetes mellitus. Diabetologia 43:1534-1539 